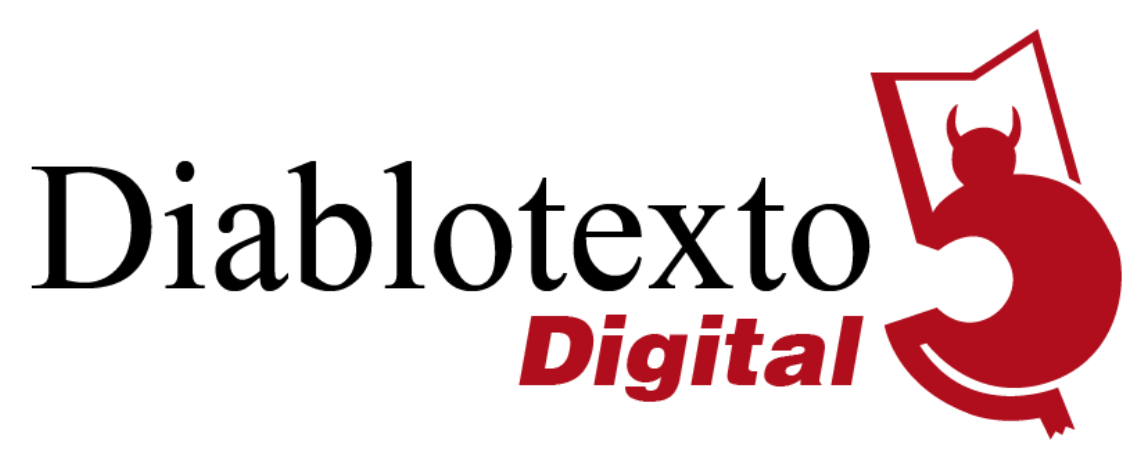

\title{
Más allá de la Realidad Virtual. Reconstrucción Acústica Virtual de antiguos teatros del Siglo de Oro. Hacia una vivencia virtual del teatro en los albores de la ultra-realidad
}

Beyond Virtual Reality. Virtual Acoustic Reconstruction of ancient theatres in the Golden Age. Towards a virtual experience of theatre at the dawn of ultrarealism

\author{
Jaume Segura Garcia, Sebastià Mirasol Menacho, Mario \\ MONTAGUD CLIMENT, JOAN OLEZA SIMÓ \\ UNIVERSITAT DE VALÈNCIA
}

\begin{abstract}
Resumen: La reconstrucción virtual de edificios antiguos y perdidos es una temática de actualidad en la conservación del patrimonio cultural. Tradicionalmente, esta reconstrucción ha sido gráfica 3D, pero si se añaden otros sentidos, la sensación de inmersión aumenta. Además, la inclusión de dimensiones emocionales y cognitivas a la percepción virtual multisensorial es lo que generará el concepto de ultra-realidad.

El objetivo de este artículo es mostrar el trabajo sobre un sistema de acústica virtual, que combina la presentación gráfica 3D y acústica (usando un motor gráfico y un motor de audio). Este sistema se implementó para renderizar auralizaciones binaurales y ha sido usado para la representación de un teatro de Valencia del siglo XVI, el llamado 'Corral de l'Olivera', y que fue construido de nuevo en 1618. Se ha modelado gráficamente y para su simulación acústica. Además, se realizó la auralización de fragmentos teatrales de diversos autores españoles. Las consecuencias de este trabajo se retornarán en futuras aportaciones sobre teatro inmersivo con mayor sensación inmersiva y emocional, contribuyendo así a la recuperación virtual inmersiva de teatros que son patrimonio cultural histórico.
\end{abstract}

Palabras clave: Realidad Virtual, Acústica Virtual, Ultra-Realidad, Auralización

Abstract: Nowadays, the virtual reconstruction of old and lost buildings is a hot-topic in cultural heritage. Traditionally, this reconstruction has been made with 3D graphical information, but the feeling of immersion can significantly increase with the addition of other senses. Moreover, the inclusion of emotional and cognitive dimensions to multisensory virtual perception is what will bring the concept of ultra-reality.

The aim of this paper is to show the work on a virtual acoustic system, which combines graphical and acoustic rendering (using a graphical engine and an audio engine). This system was implemented to render binaural auralizations and has been used for the 
rendering of a 16th century Valencian theatre, the so-called 'Corral de l'Olivera', which was rebuilt several times until the 18th century. It has been modeled graphically and for acoustic simulation. In addition, the auralization of diverse theatrical extracts of diverse Spanish authors was carried out. The consequences of this work will be reflected in future contributions on immersive theatre with greater immersive and emotional sensation. Thus, we contribute to the immersive virtual recovery of theatres that are historical cultural heritage.

Key words: Virtual Reality, Virtual Acoustics, Ultra-Reality, Auralization 


\section{Introducción}

La realidad virtual (VR) y la acústica virtual (VA) aplicada en el campo de la arqueología permite obtener reconstrucciones realistas de edificios antiguos 0 recrear estados previos de edificios actuales. Estas técnicas permiten trasladar a los usuarios a espacios de libre movimiento en el interior o en el entorno de estos edificios que se perdieron y aplicar diferentes conceptos orientados a videojuegos (gamificación), turismo virtual, etc. Entre otros ejemplos de integración de la acústica del entorno, en (Sender et al, 2017) vemos el análisis acústico y la integración gráfica de un monasterio que actualmente está en ruinas. Esta modalidad de realidad virtual permite al usuario disfrutar de una experiencia sensorial con mayor sensación inmersiva, al experimentar no solo a cómo era el edificio en diferentes épocas, sino también "catar" el sonido en el entorno como haría un catador con un buen vino. En este trabajo, analizaremos una aplicación de esta técnica a un antiguo teatro de Valencia ("Corral de Comèdies de l'Olivera") que fue derribado en 1750. Los aspectos de innovación de este trabajo se relacionan con la modularidad de los elementos de renderización acústica aplicados a la reconstrucción acústica virtual de edificios antiguos y perdidos. Por otra parte, también analizaremos posibles extensiones del mismo mediante la introducción de conceptos como multisensorialidad y ultra-realidad.

\section{El "Corral de Comèdies de l'Olivera"}

Las primeras actuaciones teatrales en València se realizaban de manera itinerante en espacios abiertos o localizaciones improvisadas sin un acondicionamiento acústico específico. El primer edificio designado específicamente para estas actuaciones lo promovió el Hospital General de València, una institución municipal y laica que tenía el monopolio de las actividades teatrales en la ciudad para financiar con sus beneficios diferentes gastos del hospital relacionados con sus actividades de caridad. El edificio ubicado en la antigua calle de la "Vall de l'Olivera" fue construido en 1584. La documentación sobre el edificio original es muy escasa, pero se conoce su localización. La vieja "Casa de l'Olivera" se mantuvo operativa durante 34 años, hasta que el Hospital decidió derribarla y construir otra nueva, con mejor ajuste 
a los requisitos funcionales de la época. Por este motivo, se adquirieron diversas propiedades anejas y se construyó la nueva "Casa de l'Olivera" (Juliá, 1950).

El nuevo edificio se construyó en el mismo lugar en 1618, con una geometría principalmente semicircular (ochavada u octogonal, más exactamente). El patio o sala tenía 20,66 metros de ancho por 19 metros de largo, permitiendo un aforo máximo de 1800 espectadores y solo en ocasiones especiales. Se distribuía en platea y dos pisos, y estaba cubierto. La documentación existente incluye estudios detallados sobre la capacidad (Mouyen, 1991a) y un plano esquemático de su planta en 1678. La platea tenía sillas con peinazo y asiento de cuero, reposabrazos y reposapiés, donde se sentaba la nobleza, los caballeros y clérigos, algunos bancos detrás de estas sillas y unas gradas de madera en la parte posterior y en los laterales. El primer piso estaba dividido en 20 palcos que eran los más lujosos y caros del teatro. Finalmente, el segundo piso, conocido como el "aposento de les dones" ("cazuela" en otros corrales españoles), estaba reservado exclusivamente a mujeres. Cada uno de estos pisos tenía su propio acceso. La figura 1 muestra la distribución de sillas en la platea del teatro. El escenario era una plataforma simple rectangular, rodeada por público en tres de sus lados. Detrás se encontraban los camerinos de los actores y sobre ellos un balcón, al cual se accedía mediante una escalera en la parte trasera y oculta del escenario, llamado "balcón de las apariciones" y que se usaba para las representaciones en un nivel superior. Esta "Casa de Comedias" se mantuvo activa hasta 1715 cuando, debido a las malas condiciones del local, se decidió abordar una remodelación significativa que se mantuvo hasta 1748, cuando el terremoto que ocasionó importantes desperfectos, unido a la prohibición de representaciones teatrales del arzobispo Andrés Mayoral Alonso de Meya ocasionó el derribo de la Casa.

Este artículo muestra la reconstrucción visual y acústica (VR/VA) de este antiguo teatro de Valencia del siglo XVII y su integración en un entorno virtual, así como las posibilidades que abre la integración de la escena en el entorno virtual y otros aspectos multisensoriales y emocionales. La integración de extractos de diferentes obras, auralizadas con las respuestas impulsivas 
obtenidas en el estudio acústico, y su orquestación/sincronización con el modelo gráfico en el entorno virtual permitirán la integración acústico/gráfica en el entorno mediante el uso de herramientas de renderización de audio espacial, como FMOD o Csound. En las páginas que siguen se detallan las propiedades, utilidades y funcionamiento de algunas de las herramientas utilizadas en el desarrollo de nuestro proyecto. El empleo de la terminología tecnológica resulta imprescindible para dar cuenta precisa de los procesos que se han desarrollado. Por ello, pese a las dificultades y hermetismo inicial que pueda comportar el uso de una terminología altamente específica, se ha apostado por su utilización desde la convicción de que compartir el metalenguaje facilita el diálogo interdisciplinar en el marco de las llamadas Humanidades Digitales. En consecuencia, se tratará de hacer una aproximación lo más diáfana posible, proporcionando las referencias necesarias para que se pueda avanzar más en este ámbito.

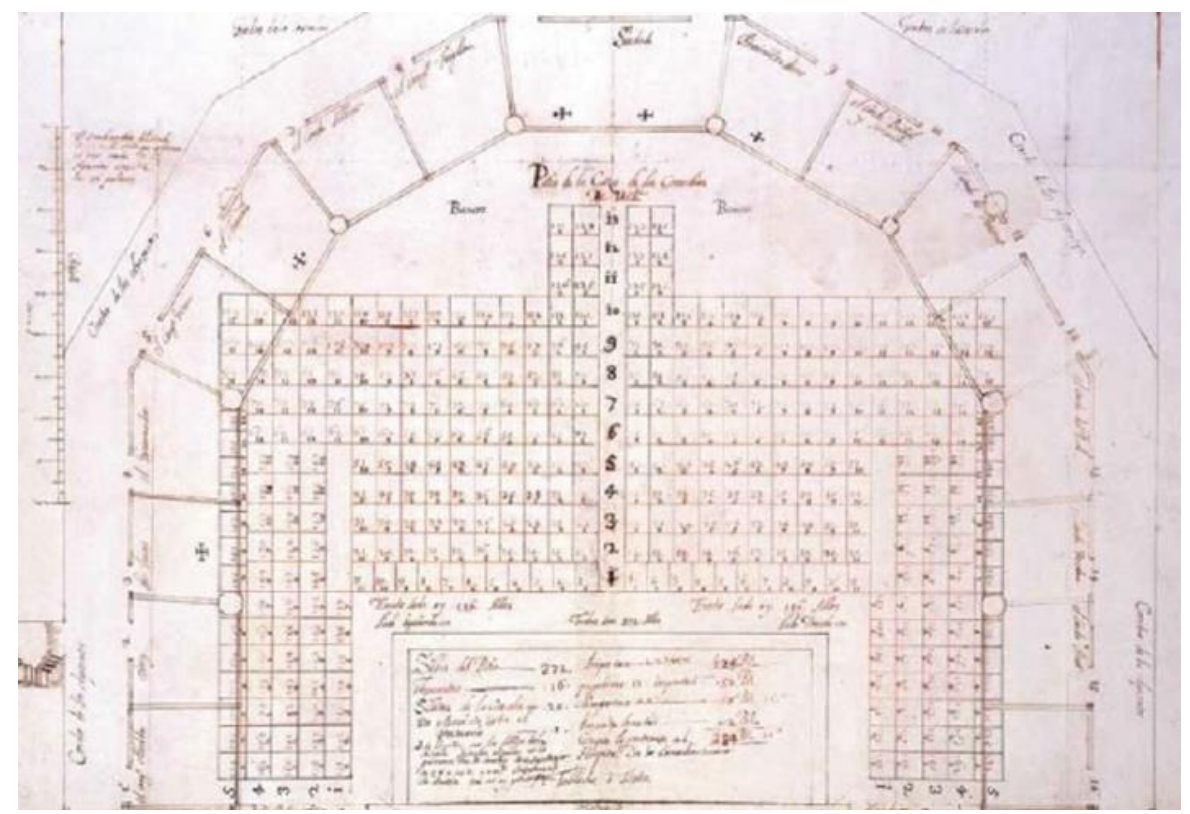

Figura 1: Plano esquemático de 1678 de la "Casa de Comedias" en València (Fuente: Archivo General y Fotográfico de la Diputación de València)

\section{Metodología}

El método de desarrollo de este trabajo se basa en la combinación de diferentes herramientas que nos permiten la integración del modelo gráfico del 
teatro y la audición de la acción teatral dentro del entorno. De esta forma, el usuario percibe una experiencia inmersiva con mayor nivel de envolvimiento a medida que la sensación multisensorial aumenta. Este estudio integrado se aplica al Corral de Comedias de "I'Olivera". Para estudiar este teatro, se ha usado como motor gráfico Unity para la reconstrucción y renderización gráfica del modelo gráfico 3D. Después del estudio acústico, las auralizaciones obtenidas de diferentes extractos teatrales se integran en motores de audio 3D, como FMOD o Csound, los cuales permiten una renderización gráfica a la vez que acústica en el entorno.

\section{Modelo gráfico y acústico}

El modelo gráfico fue construido a partir de cuatro estudios de referencia sobre este teatro: el estudio de Sirera (1980), el de Ros-Andreu (1981), el de Mouyen (1991a, 1991b) y un último de Oleza (2017), además del uso de algunos documentos antiguos que contenían información registrada para los constructores $^{1}$. El estudio de Ros-Andreu incluye planos de tres plantas, dos secciones y otra axonométrica. La figura 2 muestra estos planos. Este estudio arquitectónico fue contrastado por el profesor Joan Oleza con la documentación original de gestión y obra para los constructores, conservada en el Archivo General de la Diputación de València, y sometida a una nueva hipótesis, ahora verificada, de que la manzana correspondiente a la ubicación de este edificio no ha sufrido cambio a lo largo de la historia, por lo que las medidas actuales de la manzana nos permiten recuperar las dimensiones reales del teatro. La figura 3 verifica esta hipótesis desde el siglo XVII.

Por otra parte, la arquitecta Ana Planells re-dibujó los nuevos planos a partir de la revisión planteada por Joan Oleza y Sebastià Mirasol, siguiendo las indicaciones de Joan Oleza, montó el modelo 3D con Sketchup (Tal, 2009) para su integración en Unity. La figura 4 muestra dos capturas del modelo (también

\footnotetext{
1 "Capitulació del modo i orde que se ha de tenir en fabricar la Casa de les Comèdies conforme les traces formades per les SSo.s Administradors del Espital General". Arxiu de la Diputació de València (Julià, 1950: 46-85).
} 
se pueden visualizar dos vídeos por medio de los enlaces que se presentan en la nota al pie $^{2}$ ).

El modelo acústico se obtuvo a partir de una simplificación de este modelo gráfico, además se obtuvo el listado de materiales a partir de la documentación de los constructores (capitulaciones) con lo que se pudo estimar los coeficientes de absorción a partir de librerías de materiales. Como dejaron escrito, había seis pilares grandes que soportaban el tejado y otros cuatro más pequeños que soportaban el "ochavo" (las galerías poligonales frente al escenario) que estaban hechos de piedra de Godella, pero en el primer y segundo piso el material de los pilares cambiaba a ladrillo cubierto de yeso, igualmente que las paredes, excepto en el caso de los dos grandes pilares más cercanos al escenario, que eran enteramente de piedra. Las vigas, las cerchas, las viguetas que sustentaban los revoltons o bovedillas de los forjados, el tablado y las gradas para espectadores eran de madera. El teatro tenía suelos de loseta cerámica y barandillas de hierro en los pisos superiores.

Con el objeto de realizar el estudio acústico de este teatro, se seleccionaron las propiedades acústicas (absorción y difusión) de la base de datos de ODEON, que es el software con que se realizó la simulación acústica, ya que los materiales originales no existen actualmente y no es posible medir sus características acústicas.

Otro aspecto importante de este modelo fue la integración de la percepción acústica en el motor gráfico y algunos aspectos sobre iluminación del edificio, pero esto se tratará más tarde.

\footnotetext{
2 https://nuvol.uv.es/owncloud/index.php/s/chwXH6XotLt85Yv (trayecto en el teatro con un fragmento de "La Estrella de Sevilla" de Andres de Claramonte). https://nuvol.uv.es/owncloud/index.php/s/uk2zDNZenAQL2XF (trayecto en el teatro desde el exterior con un fragmento de "Don Gil de las calzas verdes" de Tirso de Molina).
} 


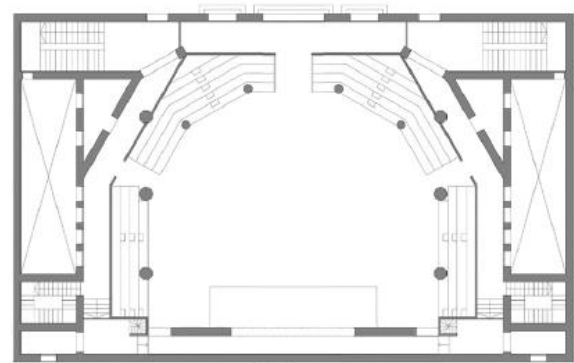

(a)

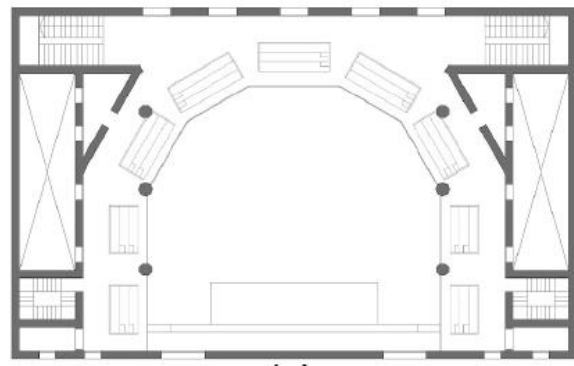

(c)

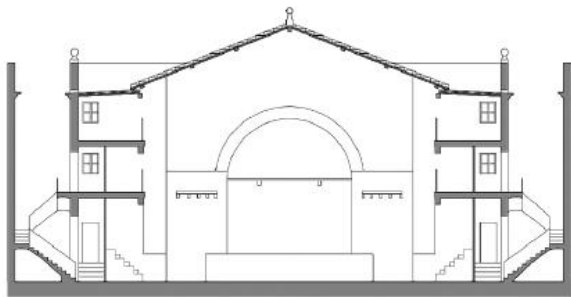

(e)

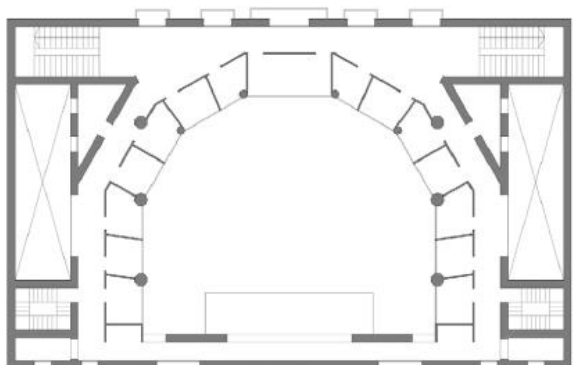

(b)

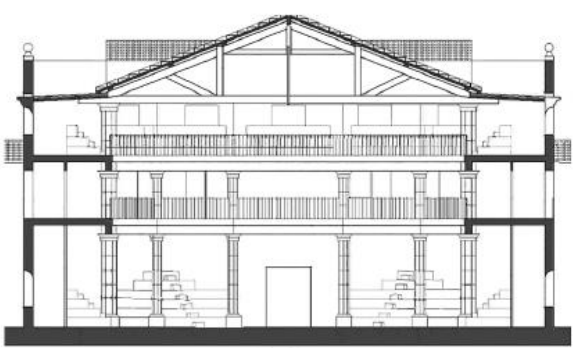

(d) fachada, (e) sección desde la fachada y (f) sección hacia el escenario

Figura 2: Planos de l'Olivera de Ros [3]: (a) planta baja, (b) primer piso, (c) segundo piso, (d)

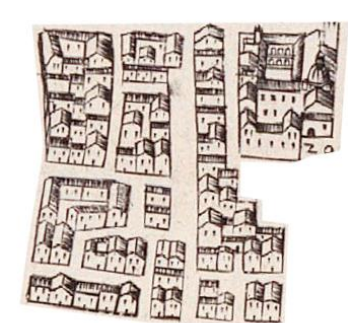

1608

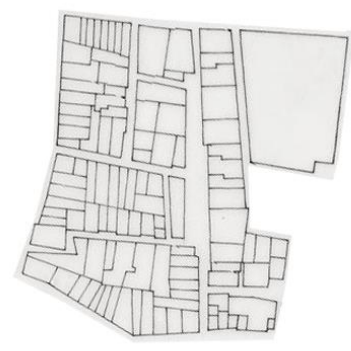

|86|

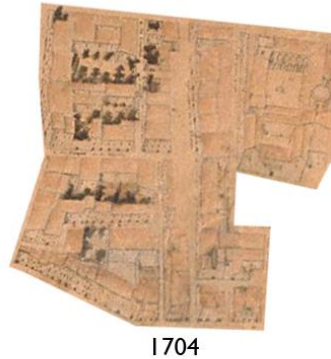

1704

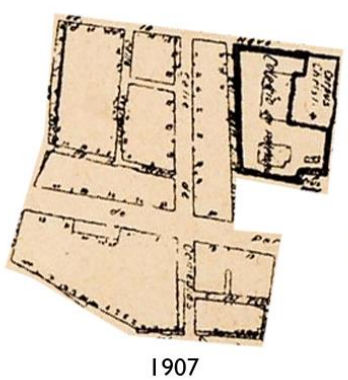

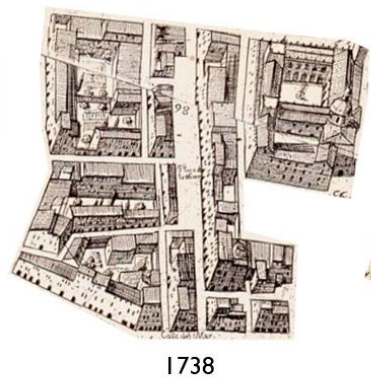

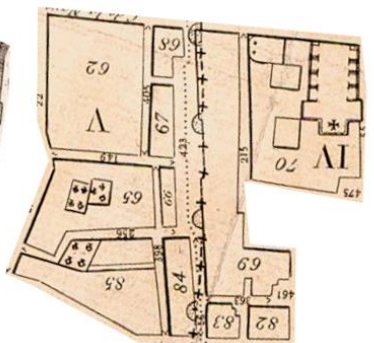

|83|
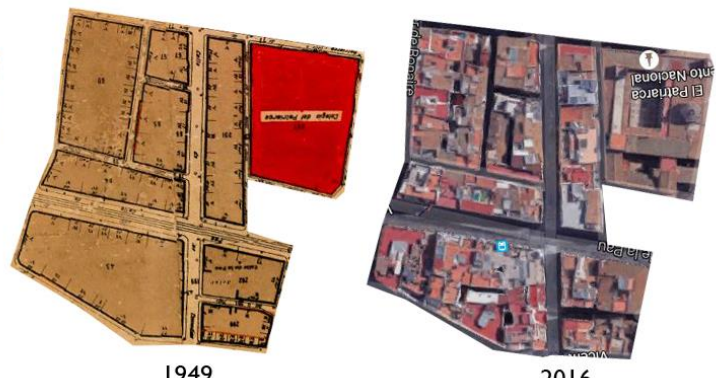

Figura 3: Comparativa de la manzana de la "Casa de l'Olivera" a partir de diferentes planos de València (Fuente: elaboración propia) 

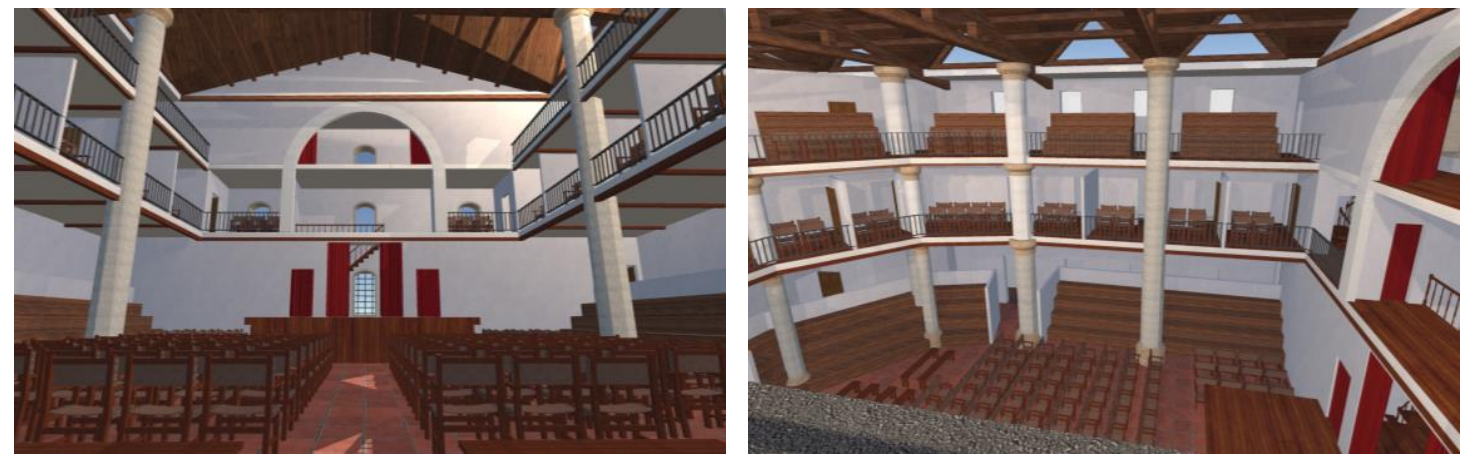

Figura 4: Modelo gráfico de l'Olivera: (a) vista desde el centro de la platea, (b) vista desde un lateral del aposento "de les dones"

\section{Grabaciones secas y auralizaciones de diferentes extractos teatrales}

Ya que este edificio se hizo en el siglo XVII, se han seleccionado algunas escenas de diversas obras de Lope de Vega para ser auralizadas ${ }^{3}$. Estas grabaciones fueron realizadas en la sala seca del laboratorio Signal Processing and Audio Technology group (SPAT) de la Escola Tècnica Superior d'Enginyeries de la Universitat de València con diversos actores profesionales de teatro clásico, como Reyes Ruiz, Enric Benavent y Toni Misò. Para ello, se usó una grabadora digital ZOOM H6 con dos micrófonos en formato XY a $90^{\circ}$ y dos micrófonos omnidireccionales adicionales para registrar las grabaciones secas para las auralizaciones.

A partir de las simulaciones acústicas, se obtuvieron las respuestas impulsivas (IRs) en diferentes posiciones. Estas ubicaciones fueron seleccionadas como referencia en cada piso. Las IRs se separan en una reverberación temprana y tardía, tomando como medida de referencia para esta sala su Early Decay Time (EDT). Estas señales pre-procesadas serán más tarde usadas para filtrar el sonido anecoico (o seco) de los extractos teatrales seleccionados.

\footnotetext{
${ }^{3}$ El concepto de auralización se define en el ámbito de la acústica como la "técnica de crear sonidos audibles a partir de datos numéricos (simulados, medidos o sintetizados)" (Vorländer, 2008).
} 


\section{Motor gráfico (Unity) y motor de audio (FMOD y Csound).}

A partir de la versión 5 de Unity, se ofrece la posibilidad de acceso a su Audio Spatializer SDK, sin embargo, aunque hace tiempo que se implementó por primera vez, este conjunto de librerías está aún muy limitado respecto a otras alternativas en el campo del audio 3D. FMOD y Csound son dos opciones que a menudo se han usado junto con Unity para realizar operaciones que el Audio Spatializer SDK de Unity no permite.

FMOD (Lahman, 2017): FMOD Studio es un entorno que permite al desarrollador el uso de una interfaz gráfica amigable para el diseño de sistemas de audio 3D mediante el uso de bloques reconfigurables (FMOD, 2018). Estos bloques permiten configurar sistemas orientados a la espacialización del sonido, que son muy útiles en entornos virtuales (ver figura 5).

Primero, se aplica un paneador 3D que separa cada canal de un sonido estéreo en dos pistas monofónicas (canal derecho e izquierdo) y permite el uso en tiempo real con diferentes paneos y distancias de atenuación. Esto es lo que permite al usuario final escuchar un sonido 3D en relación con la distancia y la dirección relativa a la fuente sonora.

Como ya hemos mencionado, el interior del edificio fue modelado acústicamente. A partir de esta simulación, se obtienen una serie de respuestas impulsivas de diferentes localizaciones interiores. Estas respuestas podrían aplicarse directamente a un reverberador convolucional, que filtra el sonido con la respuesta impulsiva sobre la marcha. Lo que hace es permitir que cualquier audio/ruido suene como si estuviese físicamente en el punto donde la respuesta impulsiva fue tomada.

Todos los cambios hechos a los sonidos se guardan en el Master Bank de FMOD. Esto es bastante útil, ya que en el futuro el usuario puede acceder a cualquiera de los sonidos independientemente, con todas las modificaciones aplicadas. 


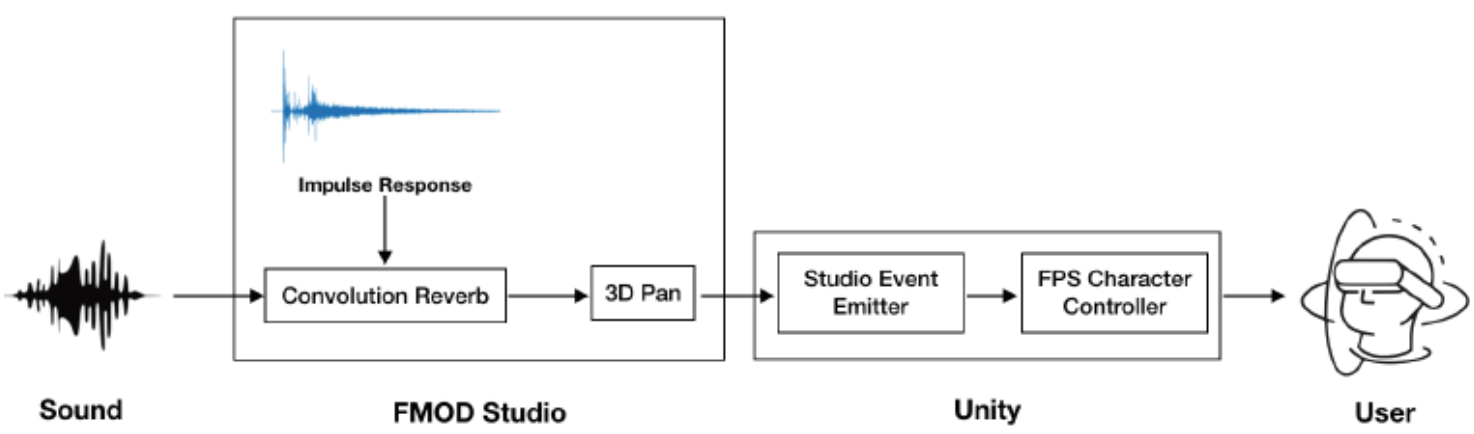

Figura 5: Esquema de módulos para la integración de FMOD con Unity

Csound (Lazzarini, 2016): es un lenguaje de programación orientado al procesado digital de señal. Desde que se lanzó en 1986, por Barry Vercoe, hasta su última versión (Csound 6) en 2013, ha habido muchas mejoras, tales como el añadido a la Application Programming Interface (API) de C, la de Python, Java, $\mathrm{C}++$ y unos cuantos lenguajes más. También ha mejorado la interpretación en vivo, permitiendo solo el score mode en tiempo real (esto significa que el uso de diferentes fuentes sonoras puede ser programado/gestionado como en una partitura). La posibilidad de programar el progreso de la señal sobre un lenguaje de alto nivel, como Python, permite una alta versatilidad en el desarrollo de sistemas. Por otra parte, la forma de orquestar procesos es a través del score mode en que las tareas se procesan en tiempo real como en un sistema de procesado paralelo.

Csound está compuesto por muchos opcodes o funciones que actúan como bloques con entradas y salidas para realizar diferentes acciones de procesado de señal. En este trabajo, se han utilizado algunos opcodes de sonido 3D binaural: hrtfmove, hrtfearly y hrtfreverb. Hay dos opcodes (hrtfearly y hrtfreverb) que permiten un modelado eficiente de las $\mathrm{HRTF}^{4}$ binaural (Carty, 2008). El opcode hrtfmove está basado en un procesado de la señal que permite el truncado de fase y obtiene la fase mínima. Por otra parte, hrtfmove usa un modelo de cabeza esférica con una menor precisión en la fase/frecuencia. Estos opcodes tienen salidas estéreo (con canales izquierdo y derecho) lo que permite

\footnotetext{
${ }^{4}$ HRTF: Head Related Transfer Function. Esta función de transferencia recoge la información de audio espacial bicanal con las características que presenta los oídos en la cabeza.
} 
usarlos con auriculares.

Cuando funciona en espacios cerrados, como el que estamos estudiando, la reverberación es un concepto clave. La figura 6 distingue entre diferentes partes de la caída energética de la respuesta impulsiva dentro de una sala y que podemos modelar con los opcodes anteriores. Hay diferentes aproximaciones para recrear una reverberación en un espacio virtual, pero Csound usa un proceso de dos etapas con los opcodes hrtfearly y hrtfreverb (Carty, 2012). El primero de ellos procesa reflexiones tempranas, emulando las reflexiones del sonido de la fuente sonora en las paredes de la sala. El segundo opcode procesa la reverberación difusa. En este caso no se trabaja directamente con las respuestas impulsivas medidas/simuladas in-situ, por lo que se necesita cierto preprocesado para obtener todos los parámetros necesarios.

El opcode hrtfearly permite al usuario usar unos pocos parámetros para ser más flexible, pero asume una geometría de sala en forma de caja de zapatos. Paralelamente a la ubicación de la fuente y el oyente en un sistema de coordenadas cartesianas, y el uso del fichero de datos de HRTFs, el usuario puede usar una sala con dimensiones por defecto o especificar la longitud, el ancho y la profundidad. Como parámetros opcionales, podemos especificar el rango de coeficientes de absorción y los factores de ganancia de un ecualizador de tres bandas, que permiten ajustar los factores de amplificación a frecuencias bajas, medias y altas.

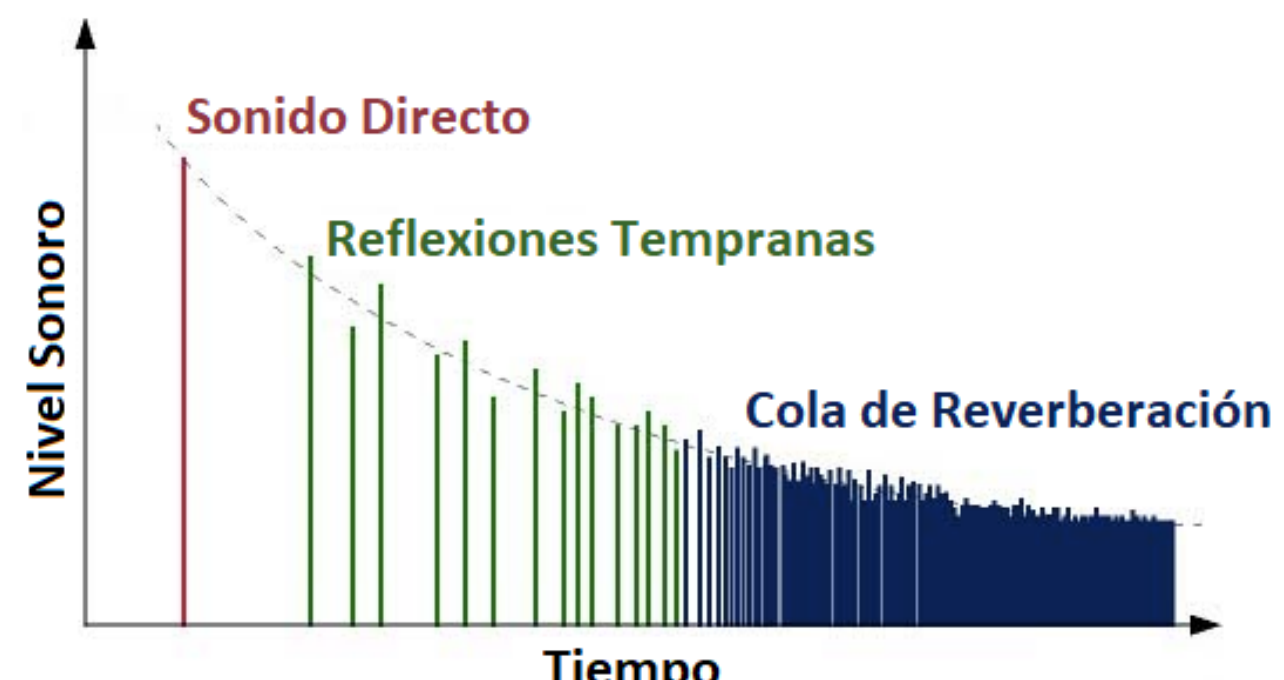

Figura 6: Diagrama de funcionamiento de la respuesta impulsiva de un entorno cerrado 
El campo difuso tardío se procesa con el opcode hrtfreverb, se puede usar independientemente o junto con hrtfearly. El uso independiente se recomienda cuando el procesado de las reflexiones tempranas es demasiado elevado. La reverberación se construye usando un paradigma de fertilización cruzada para construir la cola de reverberación tardía. Cada línea de retardo se realimenta en la anterior para añadir densidad. El algoritmo también usa filtros binaurales promediados que permite un acceso rápido a un modelo perceptivo que se aproxima al de nuestra cabeza, así como una coherencia interaural precisa, la cual permite percibir las diferencias temporales entre ambos oídos que nos dejan localizar los sonidos. El funcionamiento del opcode es más simple que el de hrtfearly, ya que solo actúa una entrada de audio y funciona para tiempos de reverberación altos y bajos. Si se necesita una reverberación más avanzada, se pueden introducir parámetros como la frecuencia de muestreo, el camino libre medio ${ }^{5}$ y el orden. Estos dos últimos parámetros se usarán para calcular un retardo adecuado para la cola tardía de la señal.

\section{Resultados}

\section{Integración de modelos gráfico y acústico}

El modelo arquitectónico del teatro perdido fue desarrollado en 3D a partir de los estudios anteriormente mencionados, usando SketchUp Pro. Después de que los expertos consideraran que el modelo era lo suficientemente preciso, se exportó a formato filmbox (.fbx) para importar después el modelo a Unity. Una vez el proceso de importación estuvo completado, y todas las texturas y materiales formateadas, procedimos a clonar el proyecto. Esto se hizo de manera que los miembros del equipo tenían dos proyectos idénticos: uno con FMOD Studio y otro con Csound.

FMOD. La integración con Unity es muy simple, los programadores ya han puesto disponible un plug-in para Unity que conecta el Master Bank de cualquier

\footnotetext{
${ }^{5}$ Distancia promedio desde la fuente sonora al receptor, recorrida por la onda sonora desde el camino recorrido por la emisión directa hasta el camino recorrido por la onda con el máximo orden de reflexión (con las paredes del entorno).
} 
proyecto de FMOD al motor de gráficos. El único ajuste necesario es asegurarse que el FPSController tiene el Audio Source deshabilitado, y el script Studio Listener de FMOD cargado como un componente. Las fuentes de audio deben hacer lo mismo, pero en vez de cargar el script Studio Event Emmitter de FMOD cabe usarlo como una componente. A partir de este momento, el usuario puede seleccionar cualquier sonido del Master Bank como fuente, con todos los faders ${ }^{6}$ que se aplicaron antes en FMOD ya aplicadas sobre la fuente sonora (ver figura 5)

Csound. Haciendo funcionar Csound con Unity es algo más complicado que FMOD. En versiones anteriores de Csound, la conexión solo podía realizarse por medio del protocolo Open Sound Control (OSC) como explica Walsh (Walsh, 2018), pero en Csound 6 se volvió inestable. El mismo Walsh desarrolló un wrapper o interfaz entre Csound y Unity (Walsh, 2014) que resultó ser la manera más fácil para realizar la interfase entre ambos motores. Después de realizar todo el preprocesado de señal con el fin de obtener todos los parámetros para los opcodes mencionados anteriormente, se implementó un instrumento para cada fuente sonora (ver figura 6). Esto limita todo el cómputo sonoro a Csound, mientras que Unity se centra únicamente en la posición de controlador y en la orientación de la cabeza que permite renderizar las HRTFs correspondientes en el formato de salida de audio adecuado.

\section{Hacia una vivencia del teatro multisensorial. El concepto de ultrarealidad}

Hasta este momento hemos considerado únicamente la inmersión acústica y visual, pero nuestro propósito es continuar nuestra investigación para la inclusión de representaciones en un entorno multisensorial. La captura de la acción teatral e incluso de los espectadores o la percepción olfativa generarán una sensación de mayor inmersión en el entorno teatral.

\footnotetext{
${ }^{6}$ Los faders son componentes que permiten la atenuación de la señal a medida que la onda se propaga.
} 


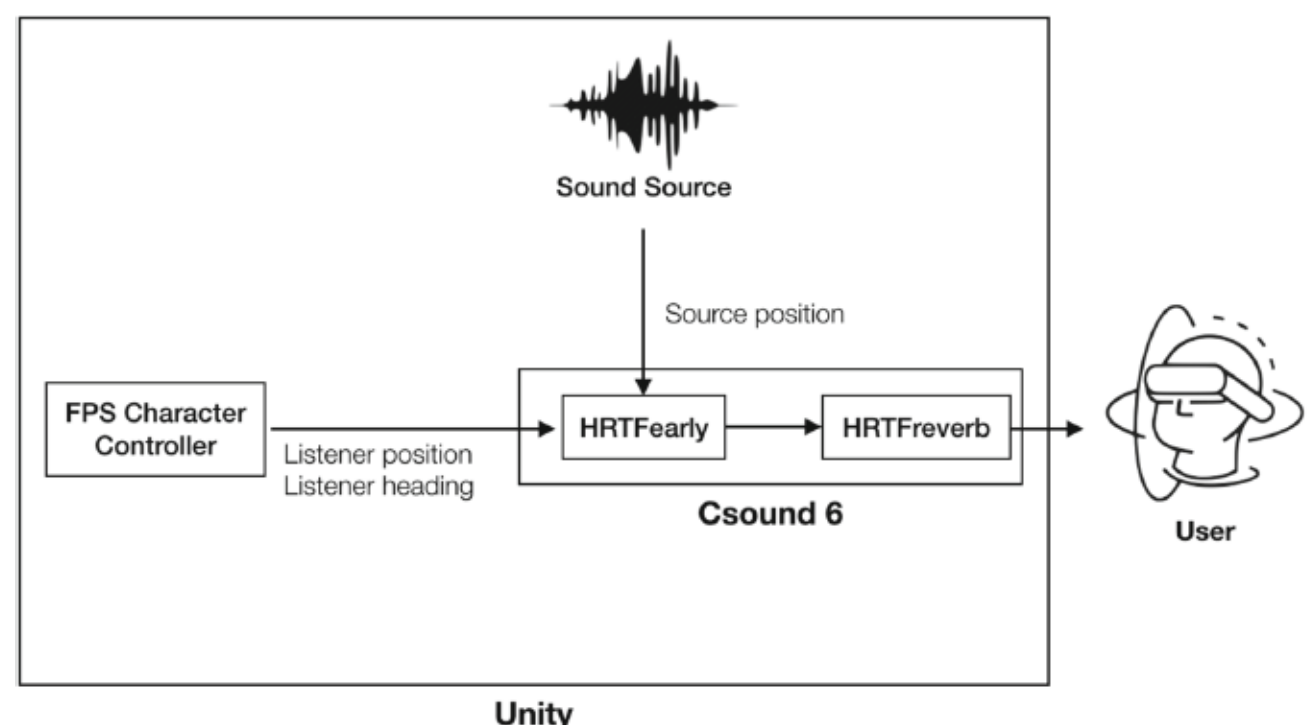

Figura 7: Esquema de módulos para integración de Csound con Unity

\section{Captura de la acción teatral}

Según el estado de la cuestión sobre dispositivos de captura tridimensional (Horaud, 2016), las cámaras de captura de profundidad son una opción muy interesante para realizar este propósito. La captura de objetos y personas es un tema de investigación que actualmente está de moda, ya que en la definición de la televisión del futuro, los investigadores tienden a incluir a los personajes e incluso los usuarios en el entorno virtual (Montagud et al., 2018a). Entre las diferentes opciones de captura se tiene el riggeado de actores animados mediante técnicas de Motion Capture (MoCap), las cámaras de profundidad (como Kinect o RealSense) y la captura de stereo billboards (mediante el uso de croma).

El uso de conjuntos de cámaras de profundidad de bajo coste, como Kinect de Microsoft Xbox o RealSense de Intel, nos permiten realizar capturas de cuerpos 3D (por ejemplo, mediante mallas o nubes de puntos) que podemos registrar e incluso integrar directamente en el motor gráfico Unity. La ubicación estratégica de diferentes cámaras de profundidad en una sala de captura de movimiento permite la integración de los actores en la escena del teatro virtual. Por otra parte, si además determinásemos la estructura esquemática del actor, podríamos incluir un avatar realizando las acciones del actor en el entorno 
virtual ${ }^{7}$.

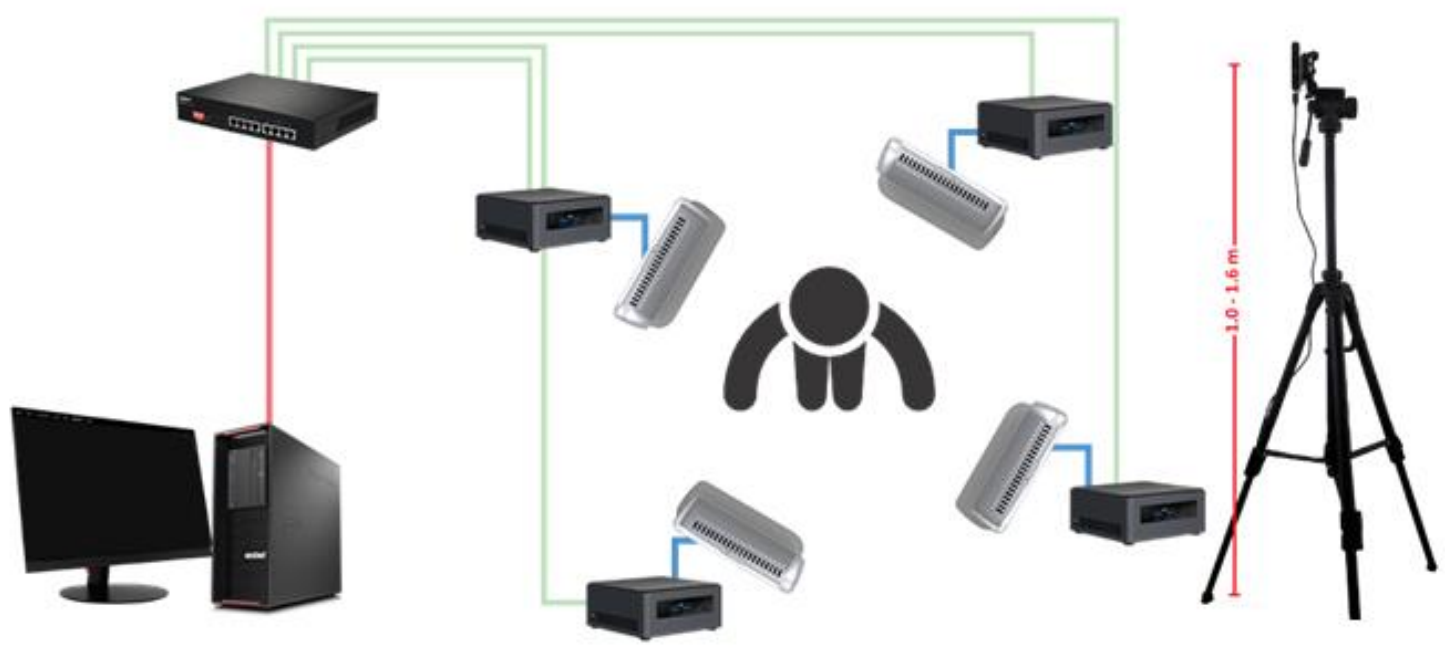

Figura 8: Esquema de un sistema de cámaras para la captura de movimiento de actores (Montagud et al., 2018b)

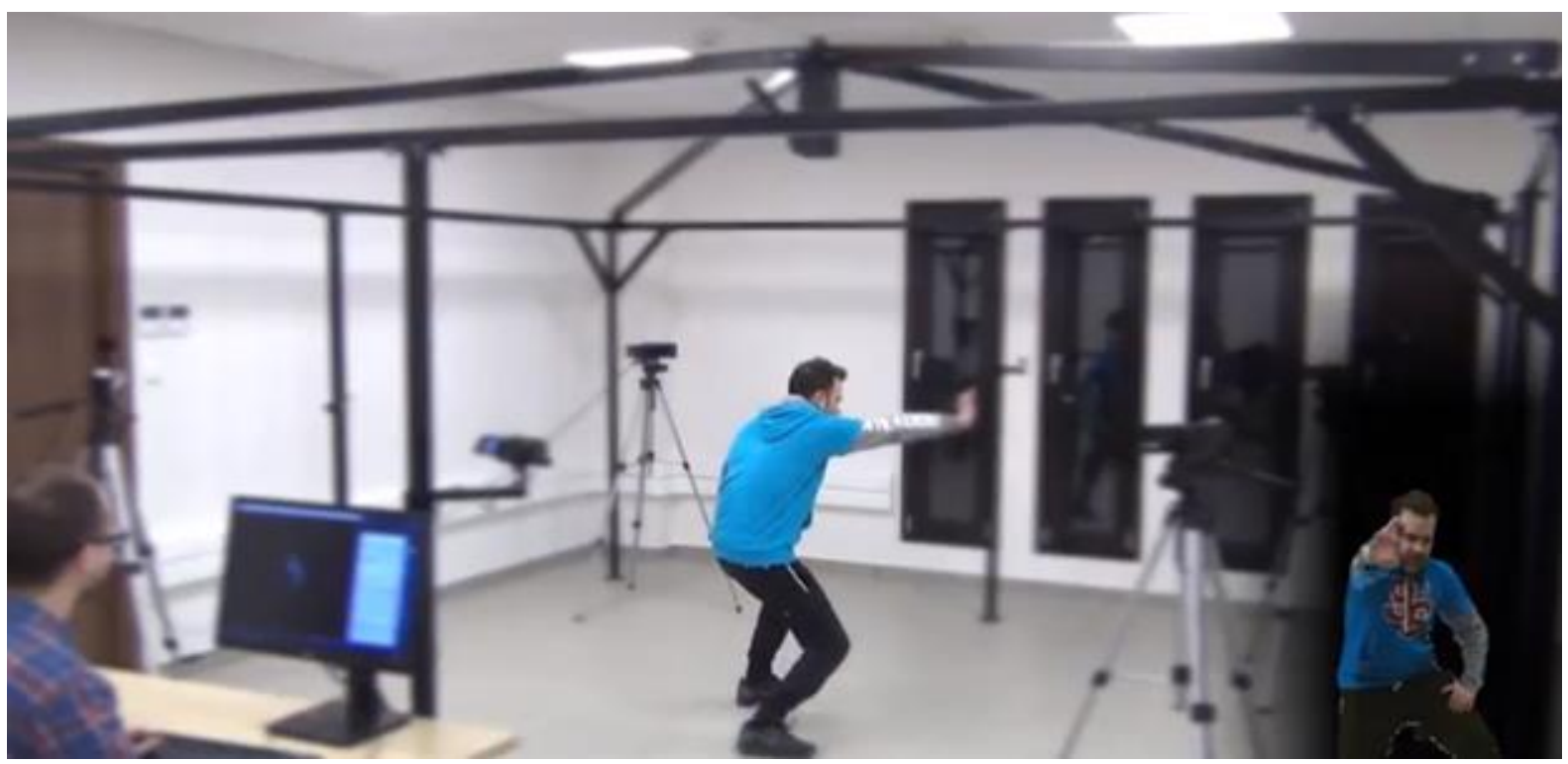

Figura 9: Fotografía de un usuario en el sistema de captura (Montagud et al., 2018b)

\section{Sensación olfativa en el teatro}

Al adentrarse en los corrales y casas de comedias del siglo XVII y una vez instalado en su localidad, el espectador debía verse envuelto en una atmósfera

${ }^{7}$ https://www.youtube.com/watch?v=WuG0egiZW2Q (visitado el 16/10/2018). 
densa, marcada intensamente por las diferentes emanaciones que afluían a sus sentidos. Es preciso imaginar que, en las condiciones de higiene de una época que no conocía el baño en las casas, ni la limpieza frecuente de la ropa, la alta concentración de personas —entre 500 y 1500 en la Casa de la Olivera de Valencia- en un espacio cerrado generaría unos efluvios olorosos considerables. A horas del mayor impacto del sol en ciudades como Madrid, Valencia o Sevilla -entre las dos o las tres y las cinco o las seis, según las estaciones, las ciudades o las fechas-. Especialmente en primavera o verano, debía proporcionar a las representaciones un ambiente impregnado de olor humano, especialmente en las localidades más propicias para la aglomeración, como las gradas de la cazuela o corredor de les dones, donde se apretujaban las mujeres, o en los patios de los corrales, donde el público de pie podía llegar a tener que estar muy apretado. Los materiales mismos del edificio contribuían a una percepción olfativa intensa: los sillones de cuero de la sala y de los aposentos en un teatro como la Olivera, la madera de las gradas, del tablado, de las cerchas y vigas de los solados y tejados, los espesos cortinajes... La cercanía de las letrinas para los espectadores, que en un teatro como el de La Olivera se situaban inmediatamente contiguas a la sala, a lado y lado, en patios muy estrechos, de apenas metro y medio de ancho - los llamados patinillos - pero que en otros teatros, como los corrales madrileños, se abrían directamente sobre los vestíbulos y bajo las escaleras de acceso, debía contribuir lo suyo a la atmósfera general. En la Casa de la Olivera, además, el foso mismo bajo el escenario, apenas separado de la sala por el tabique que servía de base al tablado, contenía un retrete casi sin ventilación para los actores. Al carecer el entorno de alcantarillado, estos edificios vertían sus aguas fecales directamente a las calles circundantes. No es extraño, por consiguiente, que los teatros dispusieran de locales dispensadores de aromas, que junto con los dispensadores de refrescos, constituían las instalaciones básicas de servicio al público. En la Casa de la Olivera, la documentación conservada alude a menudo a la existencia de una pebetería, esto es, de un local en el que se vendían o, más probablemente, alquilaban pebeteros o incensarios. Eran estos "una especie de candelabro sin candelas, en el cual se ponía el pebete en lugar de 
estas" (J. Corominas y J. A. Pascual) y el pebete era una varilla aromática "confeccionada de polvos odoríferos, que encendida echa de sí un humo muy fragante" (Covarrubias). El Diccionario de Autoridades atestigua lo aficionadas que eran las mujeres a gastar en pebetes, y estas mismas mujeres, apretadas en las gradas de la cazuela o del corredor 'de les dones', debían hacer uso frecuente de las pastillas de olores, confeccionadas "de materias aromáticas: como menjuí, estoraque, etc., que quemadas, sirven de perfume oloroso". En una ciudad como Valencia, las damas se sentían especialmente inclinadas al uso de toda clase de perfumes, y no parecía importarles demasiado su precio. Así lo dice Jaume Roig, en Lo Espill o Llibre de les dones: a las cuales "mai res los fall, /cost que costàs/.../aigües, mosquets,/ perfums, pevets,/ ab que bé olen". Y Joan Ferrandis d'Herédia, en su pieza teatral La visita, representa una divertida disputa entre una dama valenciana y su dueña castellana, en la que la valenciana hace la sátira del mal olor y la suciedad de las castellanas ('usen poc la netedat... llevan los pies "brutíssims"...'), mientras que la dueña recrimina el exceso de afeites y perfumes de las valencianas (las castellanas, dice, "traen lo sucio en los pies/, no en el rostro, como acá").

Pero más allá de la atmósfera del teatro, la representación misma aportaba numerosos estímulos sensoriales, y entre ellos los olfativos. Es el caso de las batallas, con sus descargas de mosquetería y sus cañonazos, o de las temibles tormentas, con sus truenos retumbantes, que desprendían un intenso olor a pólvora. El fuego en escena era muy frecuente, bien por requerir la acción los efectos de un incendio devastador, bien por exigir el encendido de teas y antorchas, y en ambos casos el olor a quemado parecía obligatorio, sobre todo en el caso de los incendios, que no podía representarse a la vista del espectador y debía sugerirse por los efectos concomitantes. En ocasiones, hacían su aparición espectacular jinetes sobre sus cabalgaduras, que daban la vuelta al teatro, y que podían llegar a subir al escenario por rampas especialmente dispuestas. Las apariciones sulfurosas de las furias infernales en las comedias mitológicas o los descensos a los infiernos y ascensos al cielo de condenados y bienaventurados en las comedias de santos requerían efectos especiales que probablemente comportaban efluvios olorosos. Y si nos situamos más en la 
percepción del actor que en la de los espectadores, entonces todo el cortejo amoroso de las comedias urbanas debía acompañarse de sensaciones estimulantes y aromáticas. En una obra como La viuda valenciana, la protagonista atrae cada noche a su casa al galán del que anda enamorada, con la condición de que se deje encapuchar y tapar los ojos, para no ver ni el lugar donde vive, ni reconocer la identidad de la dama. El galán se verá entonces sometido a una seducción a oscuras, en la que la belleza de su amada y la sofisticación de su entorno doméstico, así como la atención cómplice de los criados, encenderán su deseo no por la vista sino por la música, los aromas, y las caricias. Es solo una muestra de hasta qué punto, sobre la escena, se podría jugar con las percepciones sensoriales de actores y espectadores.

La determinación de este ambiente permite recoger un conjunto de esencias sintetizadas para poder difundirlas de manera orquestada dentro del entorno. Una posible forma de sintetizar estas esencias, de manera segura, sería mediante el uso de levaduras de tipo alimentario derivadas de la Saccharomyces cerevisiae. Esta puede ser utilizada como una fábrica de células microbianas para la producción de olores y componentes aromáticos, proporcionando así una forma de producción sostenible y eficaz de sustancias químicas que normalmente son raras y a menudo costosas. La biología sintética es una de las ramas de la biología que más rápidamente evoluciona, ya que permite la introducción de vías genéticas personalizadas en organismos que les proporcionan la capacidad de mostrar propiedades biológicas y/o bioquímicas por encima de un fondo original, a menudo silvestre. Recientemente se ha reportado numerosos ejemplos de implementación de biología sintética con S.cerevisiae, como por ejemplo en la producción de compuestos biomédicos, biocombustibles (Turner et al., 2018) o diferentes aromas en vino (Liu et al., 2018) y cerveza (Capece et al, 2018).

El uso de difusores de olores nos permitirá seleccionar y combinar un conjunto de esencias que se presentan en este tipo de ambiente a partir de la literatura de la época (por ejemplo, sudor, incienso, orina, etc.), con una alineación espacial y temporal con el entorno virtual, y con unos niveles de intensidad ajustables. En nuestro entorno podemos encontrar algunas 
compañías dedicadas a la creación de esencias e incluso con aplicaciones tecnológicas (VR/AR). Es el caso de Olorama, una pequeña empresa tecnológica de Torrent (Valencia), que además de un conjunto de aromas, ofrece dispositivos difusores y una $\mathrm{SDK}^{8}$ para interactuar con sistemas de realidad virtual o aumentada. Por otra parte, la inclusión de olores adecuados (y contextualizados) en los entornos virtuales, además de producir un grado de realismo en la escena, ocasionando mayor sensación de inmersividad, tienen un impacto emocional y actitudinal en los usuarios, por lo que resultan una herramienta útil para "enganchar" al espectador y captar su atención en determinados momentos (Obrist et al., 2014).

\section{El concepto de ultra-realidad}

Este concepto (Ikey et al., 2012) fue acuñado en 2005 por un comité gubernamental japonés que exploraba fuentes de innovación en tecnologías de la información y la comunicación. En 2007, se estableció un foro conjunto entre entidades gubernamentales, industriales y académicas (Ultra-Realistic Communications Forum) que empezó la exploración del concepto enfocándolo en tres aspectos: vídeo/imagen estéreo en muy alta definición (UHD), generación sonora realista y comunicaciones multisensoriales. La comunidad científica incluyó entre estos temas el de la sensación de presencia inducida en nuestros sentidos de forma artificial por medios tecnológicos. Por otra parte, el mecanismo psicológico de la sensación de presencia no ha sido completamente investigado para proporcionar una base bien establecida sobre la que construir un sistema que permita crear de forma efectiva un generador de presencia. En (Andoh, 2010), se proponen tres factores que constituyen la sensación de presencia: el factor espacial (sensación de estereognosis, textura e inmersión), el factor temporal (sensación de movimiento y sincronía) y el factor corporal (sensación de autoexistencia, percepción de causalidad, de interactividad y emocional). Además, las fuentes de sensación de presencia son externas (perceptuales) e

\footnotetext{
${ }^{8}$ SDK (Software Development Kit): es un conjunto de librerías y programas que permiten el desarrollo de aplicaciones más complejas y para controlar diferentes elementos hardware y software. También permite elementos de interacción.
} 
internas (memoria), por lo que la interpretación de este concepto va más allá de la construcción tecnológica que establecería los límites sensoriales del sistema de realidad virtual, entrando en un campo de percepción subjetiva.

Creemos que la aplicación de este concepto al campo teatral, permitirá una nueva forma de entender el fenómeno social que constituye esta actividad social, si se genera la tecnología adecuada para recrear el entorno social dentro del mundo virtual, al mismo tiempo que se permitan la interacción entre los usuarios, y entre los usuarios y el mismo entorno virtual. Esta es una de las líneas de investigación que queremos promover en la Universitat de València. Además, esto abrirá muchas posibilidades en la industria multimedia en general (por ejemplo, en el campo de la televisión interactiva o el turismo virtual), mediante la creación de contenidos combinando tecnología y humanidades digitales.

\section{Glosario de términos técnicos}

Auralización: técnica de procesado de señal que permite crear sonidos audibles a partir de datos numéricos (simulados, medidos o sintetizados) (Vorländer, 2008).

Bloque reconfigurable: Conjunto funcional, en un entorno de programación o programa software específico, con características configurables que permite aplicar una serie de operaciones a una entrada (p. e. señal, mensaje...) para, a la salida, obtener un resultado diferente.

Estereognosis: reconocimiento de objetos mediante la percepción de su forma, volumen y consistencia a través del tacto.

Gamificación: es el uso de estrategias, modelos, dinámicas, mecánicas y elementos propios de los juegos en contextos ajenos a éstos, con el fin de transmitir un mensaje o unos contenidos o de cambiar un comportamiento, a través de una experiencia lúdica que propicie la motivación, la implicación y la diversión. Sin embargo, es importante tener en cuenta que la información transmitida en estos casos suele ser de carácter práctico principalmente.

Grabación seca/anecoica: la grabación seca se refiere al registro sonoro de fuentes en entornos con baja o muy baja reverberación (0,2-0,4 s) y próximo a la fuente, mientras que la grabación anecoica es aquel registro sonoro que se 
realiza en una cámara anecoica donde no hay reverberación (simula el campo libre, donde cualquier onda sonora emitida no puede reflejarse en ninguna superficie y es atenuada a medida que se propaga).

Paneador (o "panoramizador"): es una técnica para regular el envío de señales sonoras en un medio estéreo o multicanal con más intensidad por unos canales que por otros para crear una imagen sonora de la ubicación de cada fuente.

Renderización: término en jerga informática para referirse al proceso de generación de una imagen o sonido en un determinado entorno a partir de información digital (normalmente a partir de una simulación). En ocasiones también se refiere a la presentación de esta imagen o sonido en ese entorno simulado.

Reverberador convolucional: es un tipo especial de filtrado que se realiza al filtrar o convolucionar un sonido anecoico con una señal que representa la respuesta al impulso de una determinada sala. En sistemas de procesado digital de señal (DSPs) pueden configurarse a partir de ciertos parámetros característicos de la sala (p. e. tiempo de reverberación).

Rigggeado: en el ámbito de la animación, es el proceso de creación del esqueleto o estructura simplificada del actor o avatar que actúa en el entorno virtual.

Sonido binaural: es aquel audio registrado mediante el uso de micrófonos en los oídos de una cabeza artificial (o natural), para tratar de crear en el oyente una sensación de sonido 3D similar a la que se produce en el lugar donde se produjeron los sonidos.

\section{BiBLIOGRAFÍA}

ANDOH, H. (2010). "Factors of the sense of presence". En Tachi, Sato \& Hirose (eds.), Virtual Reality, 6.2.2. VRSJ.

BARBA, A., GiméneZ, A. (2011). El teatro principal de Valencia: acústica y arquitectura escénica. Valencia: Generalitat Valenciana, Universitat Politècnica de València.

Capece, A.; Romaniello, R.; Pietrafesa, A.; Siesto, G.; Pietrafesa, R.; ZAMBUTO, M.; ROMANO, P. (2018). "Use of Saccharomyces cerevisiae var. boulardii in co-fermentations with $\mathrm{S}$. cerevisiae for the production of craft beers with potential healthy value-added". International Journal of Food Microbiology, 2018, 284, pp. 22-30. DOI: https://doi.org/10.1016/j.ijfoodmicro.2018.06.028

CARTY, B. (2008). "HRTFmove, HRTFstat, HRTFmove2: using the new HRTF 
Opcodes". Csound J., 9.

CHRISTENSEN CL AND KOUTSOURIS G. (2015). ODEON room acoustic software, version 13, full user's manual. Kongens Lyngby: Odeon A/S, Schion DTU.

COROMINAS, J. y PASCUAL, J. A. (1991-1997). Diccionario crítico etimológico castellano e hispánico. Madrid, Gredos, 6 vols.

CovarRUBIAS, Sebastián de (2006). Tesoro de la lengua castellana o española. Ed. de I. Arellano y R. Zafra. Madrid: Iberoamericana-Vervuert.

Diccionario de Autoridades [1726-1739] (1963). Real Academia Española, Madrid: Gredos.

FERRANDIS D'HERĖDIA, Joan (1955). La vesita o Coloquio en el cual se remeda el uso, trato y pláticas que las damas de Valencia acostumbran hacer y tener en las visitas (1524), en Juan Fernández de Heredia, Obras. Ed. de R. Ferreres. Madrid: Clásicos Castellanos.

Horaud, R., Hansard, M., Evangelidis, G., Ménier, C. (2016). "An Overview of Depth Cameras and Range Scanners Based on Time-of-Flight Technologies". Machine Vision and Applications Journal, 27 (7), pp. 10051020.

Ikel, Y., Abe, K. HiRota, K., AmemiYa, T. (2012). "A multisensory VR system exploring the ultra-reality," 2012 18th International Conference on Virtual Systems and Multimedia, Milán, pp. 71-78.

JULIÁ, E. (1950). "Nuevos datos sobre la Casa de la Olivera de Valencia", Boletín de la Real Academia Española, 30, pp. 47-85.

Lanham, M. (2017). Game Audio Development with Unity 5.X. Packt Publishing. https://www.safaribooksonline.com/library/view/game-audio development/9781787286450/. [Fecha de consulta: 10 de marzo de 2018].

LazZarinI, V., YI, S., FFITCH, J., HeintZ, J., BRANDTSEgG, Ø., McCuRdy, I. (2016). Csound: A Sound and Music Computing System. Springer, Heidelberg. DOI: 10.1007/978-3-319-45370-5

LIU, P.-T., YU, K.J., LI, Y.T., DUAN, C.Q., YAN, G.L. (2018). "The content of linoleic acid in grape must influences the aromatic effect of branched-chain amino acids addition on red wine". Food Research International, 114, pp. 214222. DOI: 10.1016/j.foodres.2018.08.016

Montagud, M., NúÑ̃EZ, J. A., Karavellas, T., JuRado, I., Fernández, S. (2018). "Convergence between TV and VR: Enabling Truly Immersive and Social Experiences". Workshop on Virtual Reality, co-located with ACM TVX 2018, Seoul (South Korea), June. DOI: https://doi.org/10.6084/m9.figshare.6526214.v1

Montagud, M., NúÑez, J. A., Fernández, S., Orero P., Matamala, A. (2018). "ImAc: Enabling Immersive and Accessible Media Services". Workshop on Reading, Language \& Deafness. Research perspectives. San Sebastián, Spain, Oct 18-19

MOUYEN, J. (1991). "El corral de la Olivera de Valencia en 1678 y 1682: tentativa de definición sociológica de su público". En M. V. Diago y T. Ferrer (eds.), Comedias y comediantes. Valencia: Universidad de Valencia, 1991, pp. 407-432

MOUYEN, J. (1991). "Las casas de comedias de Valencia". Cuadernos de Teatro Clásico, 6, pp. 91-122. 
ObRIST, M., TUCH, A. N and HORNB/EK, K. (2014). "Opportunities for odor: experiences with smell and implications for technology". CHI 2014 Conference on Human Factors in Computing Systems, Toronto, Canada, April 26 - May 01. DOI: https://doi.org/10.1145/2556288.2557008

OlEZA, J. (2017). "La casa de comedias de La Olivera. Reconstrucción virtual de un teatro distinto". XVIII Congreso Internacional de la AITENSO. Universidad Iberoamericana \& El Colegio de México, México, 23-27 de octubre (en prensa).

RoIG, Jaume [1460?] (1978). Espill o Llibre de les dones. A cura de M. Gustà, pròleg de J. Bergés. Barcelona: Edicions 62 i La Caixa.

Ros ANDREU, J. LI. (1981). L'Efímer en la formació del Barroc valencià, 15991632. Tesis doctoral, Universitat Politècnica de València.

Sender, M.; Planells, A.; Perelló, R.; Segura, J.; Giménez, A. (2017). "Virtual acoustic reconstruction of a lost church: application to an Order of Saint Jerome monastery in Alzira, Spain". Journal of Building Performance Simulation 11(3), pp. 369-390. DOI: https://doi.org/10.1080/19401493.2017.1340975

SIRERA, J. L. (1980). El teatro en Valencia durante los siglos XVI y XVII: la producción dramática valenciana en los orígenes de la comedia barroca. Tesis doctoral, Universidad de Valencia.

TAL, D. (2009). Google SketchUp for Site Design: A Guide to Modeling Site Plans, Terrain and Architecture. Series CourseSmart, Wiley.

TURNER, T.L.K.H., KONG, I.I., LIU, J.J., ZHANG, G.C., JIN, Y.S. (2018). Engineering and Evolution of Saccharomyces cerevisiae to Produce Biofuels and Chemicals; Cham, Switzerland: Springer International Publishing, pp. 175215.

VORLÄNDER, M. (2008). Auralization: Fundamentals of Acoustics, Modelling, Simulation, Algorithms and Acoustic Virtual Reality. Berlin: Springer.

Getting Started with FMOD in Unity. https://www.fmod.com/api. [Fecha de consulta: 15 de febrero de 2018].

Studio 3D Events.

https://www.fmod.org/docs/content/generated/overview/3dstudio.html. [Fecha de consulta: 16 de febrero de 2018].

Fecha de recepción: 15 de noviembre de 2018

Fecha de aceptación: 30 de diciembre de 2018 